Изв. Крымск. Астрофиз. Обсерв. 116, № 1, 14-23 (2020) doi:10.31059/izcrao-vol116-iss1-pp14-23

УДК 523.9

\title{
Крымские наблюдения магнитного Солнца: 1967-2018 гг.
}

\author{
B.A. Komoв \\ ФГБУН “Крымская астрофизическая обсерватория РАН", Научный, Крым, 298409 \\ vkotov@craocrimea.ru
}

Поступила в редакцию 17 июня 2020 г.

\begin{abstract}
Аннотация. Измерения магнитного поля Солнца как звезды, начатые по инициативе акад. А.Б. Северного, были поддержаны шестью другими обсерваториями. Кратко изложены история таких исследований в Крымской астрофизической обсерватории и основные результаты. По вариациям поля определен синодический период вращения солнечной гравитирующей массы $P_{\odot}=27.027(6)$ сут. Обнаружено, что к нему привязаны движения Земли: за один земной год Солнце совершает 27 полуоборотов вокруг своей оси, а Земля - такое же число своих вращений с периодом $P_{D}$ за полный солнечный оборот. Поле изменяется также с циклом Хейла $P_{H} \approx 22$ г. и циклом $P_{7} \approx 7$ лет, причем их отношение совпадает с приближением Архимеда, 22:7, для числа $\pi$, а временна́я шкала $(\pi-3) P_{7}=P_{\odot}^{2} / 2 P_{D}-$ с орбитальным периодом Земли. Приведены аргументы в пользу космической природы обоих циклов и голографические выражения с участием $P_{H}, P_{7}, \pi$ и универсальных констант.
\end{abstract}

Ключевые слова: история КрАО, Солнце, магнитное поле, вращение, 11-летний цикл

\section{1 Введение}

В течение более полувека в Крымской астрофизической обсерватории (КрАО) осуществлялся уникальный эксперимент: регулярные измерения эффекта Зеемана фотосферы Солнца, наблюдаемого как звезда, по эффекту Зеемана спектральных линий поглощения фотосферы. Измерения проводились на телескопе БСТ-1 с помощью инструмента типа магнитографа Бэбкока - крымского солнечного магнитографа, созданного техническим дарованием Н.С. Никулина по идеям и при участии А.Б. Северного и В.Е. Степанова (Никулин и др., 1958). Такие наблюдения Солнца в 1970-2020 гг. были поддержаны шестью другими обсерваториями мира (см. п. 3).

\section{2 БСТ-1}

Главными задачами Башенного солнечного телескопа (БСТ-1, до и/или после реконструкции, осуществленной в 1970-1973 гг. под руководством А.Г. Перегуды и Г.А. Монина; см. Северный, 1955; Котов и др., 1982) были: (1) получение спектрограмм высокого пространственного и спектрального разрешения (фотосферы, пятен, вспышек, выбросов вещества, протуберанцев и других образований на Солнце); (2) зарисовки пятен и визуальное - с помощью поляроидной мозаики - измерение максимальной абсолютной напряженности магнитного поля (м. п.) пятен; (3) фотографирование диска Солнца в лучах линий $H_{\alpha}$ водорода и К Ca II на фотопластинках с помощью двухлучевого спектрогелиографа; (4) измерение м. п. по различным спектральным линиям; (5) измерение путем многочасового сканирования м. п. всего диска, а также полей полюсов в спокойных и активных областях (а. о.), в пятнах и протуберанцах; (6) измерение полного вектора м. п. в пятнах и а. о.; (7) исследование тонкой структуры и вариаций м. п., яркости и лучевой скорости (спокойной и активной фотосферы, ее различных деталей - с разрешением до $1^{\prime \prime}$ ); (8) прогноз вспышечной активности ("программа Северного" 60-х годов: по магнитограмме а. о. определялись градиенты м. п. и структура электрических токов, а по структуре, с привлечением наблюдений на коронографе 
КГ-1, - вероятность сильных вспышек в течение предстоящих трех суток; делалось это перед и во время полетов космонавтов с целью предупреждения о радиационной опасности в космосе; оправдываемость 70\%); (9) с 1974 г. - наблюдения глобальных пульсаций Солнца (Severny et al., 1976; Котов, Ханейчук, 2016).

Были и такие эпизодические работы, как (a) спектральные наблюдения на вакуумном спектрографе (получение спектров высокого качества), (б) регистрация локальных акустических (“5минутных") колебаний лучевой скорости фотосферы, (в) инфракрасные (ИК) наблюдения, в частности, измерения потемнения диска к краю, проведенные совместно с Институтом астрофизики в Париже (ИАП), (г) регистрация глобальных колебаний яркости Солнца с применением магнитографа или (д) механического ИК-модулятора (с ИАП), а потом (е) фотодиодных матриц, и (ж) регистрация глобальных колебаний общего магнитного поля (ОМП) Солнца.

После 1994 г. группа Э. Родса в течение нескольких лет исследовала акустические колебания фотосферы с применением магнито-оптического фильтра. Полученные данные отправлялись в США, в частности, для корректировки по пятнам и ориентации прибора $M D I$ на спутнике $S O H O$. В то же время группа И.А. Егановой (Новосибирск) проводила работу по проверке гипотетических эффектов Н.А. Козырева с регистрацией изменения массы минерала со временем под воздействием "внешних необратимых процессов".

В наблюдениях на БСТ-1 по различным программам и в разные годы (1965-2017 гг.) участвовали: А.Б. Северный, С.И. Гопасюк, В.И. Ханейчук, Т.Т. Цап, В.А. Котов, а также А.С. Андреев, О.А. Андреева, А.Н. Бабин, Э.А. Барановский, О.С. Гопасюк, М.Дж. Гусейнов, М.Л. Демидов, Л.В. Дидковский, А.В. Долгушин, И.А. Еганова, Д.И. Иргашев, Р.Н. Ихсанов, Б. Калман (Венгрия), А.Н. Коваль, В.М. Кувшинов, Л. Кулчар (Чехословакия), С. Кучми (Франция), Ли Руфень (КНР), Лыу Ван Лыонг (ДРВ), В.П. Маланушенко, С.И. Плачинда, Д.Н. Рачковский, Э. Родс (США), Я. Стенфло (Швеция), Н.Н. Степанян, Н.В. Стешенко, Ф. Шеррер (США), А.Г. Щербаков, В.Б. Юрчишин. Техническое обеспечение работы телескопа и инструментов осуществляли: Н.С. Никулин, А.М. Чижов, Л.Ф. Бежко, Л.В. Дидковский, И.П. Залесов, В.И. Лопухин, В.И. Ханейчук, а также: А.В. Брунс, А.В. Долгополов, А.Р. Пулатов, Н.П. Русак, Д.Г. Семёнов, Л.Н. Стуков, Г.А. Суница и др., включая работников мастерских КрАО; автоматизация, компьютерное и программное обеспечение: Н.С. Никулин, А.В. Букач, Л.А. Границкая, Л.В. Дидковский, И.П. Залесов и В.И. Ханейчук. Далее приводится хронология наблюдений Солнца как звезды (см. также Котов, 2007).

\section{3 Краткая история}

В ноябре 1967 г. Андрей Борисович Северный пришел на телескоп и предложил мне измерить эффект Зеемана всего диска Солнца без пространственного разрешения. (Он как раз вернулся с международного симпозиума, где с Р. Говардом и В. Бумбой обсуждал возможность измерений поля Солнца как звезды. Говард для этого усреднял магнитограммы диска, полученные им в обсерватории Маунт Уилсон, но из-за неопределенности "нуля" магнитографа, погрешностей калибровки и других эффектов ошибки составляли несколько гаусс, так что искомый сигнал тонул в шумах.)

Мы с Северным сразу же направили пучок солнечного света от целостатных зеркал на диагональное зеркало, а от него - на входную щель спектрографа (в таких наблюдениях участвуют только плоские зеркала: Солнце наблюдается как звезда, или, как мы говорили, в “параллельном пучке": щель спектрографа освещается всем солнечным диском; измеряется степень круговой поляризации в крыльях спектральной линии, чувствительной к м. п.). Если эффект Зеемана обычно на БСТ-1 регистрировался по линии поглощения Fе I $\lambda 525.0$ нм, с фактором Ланде $g=3$, то "нуль" магнитографа Северный предложил фиксировать по аналогичному сигналу, полученному по линии Fe I $\lambda 512.4$ нм, $g=0$. Первая же запись длительностью 20 мин для каждой линии показала, что ОМП (продольная компонента) измеряется с ошибкой 0.10-0.15 Гс, причем с известным, почти идеальным "нулем" инструмента! Такие измерения регулярно проводились на БСТ-1 в 1968-1969 гг., а с 1970 г. по 1976 г., в связи с реконструкцией БСТ-1, - на телескопе БСТ-2; в 1991 г. были возобновлены на БСТ-1, ныне им. акад. А.Б. Северного.

С 1967 г. в наблюдениях ОМП в КрАО в разные годы участвовали: А.Б. Северный, С.И. Гопасюк, В.И. Ханейчук, Т.Т. Цап, В.А. Котов, а также М.Дж. Гусейнов, М.Л. Демидов, Д.И. Иргашев и С.И. Плачинда; в обработке наблюдательного материала: А.Б. Северный, С.А. Бондаренко, 
Е.И. Лиморенко, Г.Я. Смирнова, Н.Г. Суница, Н.П. Фролова, А.В. Ханейчук, В.И. Ханейчук, Н.Ф. Черных и В.А. Котов.

После первого года наблюдений вышла в свет работа Severny (1969) с главными выводами: (a) ОМП реально измеряется, (б) оно имеет секторную структуру (с признаками двух и четырех секторов одной полярности на один солнечный оборот; потом было показано, что секторная структура ОМП согласуется с таковой межпланетного поля; Scherrer et al., 1977a), (в) суточные значения поля в 1968 г. изменялись в пределах от -1.5 Гс до +0.8 Гс, (2) поля пятен не вносят существенного вклада в результирующий сигнал ОМП и (д) само Солнце - магнитный ротатор с синодическим периодом вращения $\approx 27$ сут. (Подобные наблюдения Северным и др. (1974) были начаты позднее на телескопе 3ТШ для измерения слабых магнитных полей звезд.)

В 1970 г., благодаря новизне этой работы, КрАО посетил Дж. Уилкокс (Стэнфордский университет, США); в том же году по его инициативе Р. Говард и Ф. Шеррер начали измерять ОМП в обсерватории Маунт Уилсон, а в 1971 г. Северный с супругой были гостями Уилкокса. Все это послужило импульсом к созданию в 1974 г. в Стэнфорде специальной обсерватории (ныне Солнечная обсерватория им. Дж. Уилкокса, WSO) для исследований ОМП, полярного поля и крупномасштабных полей на Солнце. В состав обсерватории входил башенный телескоп, целостатная установка, вертикальный спектрограф, снабженный светоделителем, и регистрирующий инструмент типа магнитографа Бэбкока. В связи с этим строительством я посетил Стэнфорд в 1974 г., а Шеррер с супругой - КрАО в 1975 г.

Крымские измерения 1968-1976 гг. составили первый в мире каталог ОМП (Котов, Северный, 1983). Ниже приведены некоторые результаты таких исследований в КрАО.

\section{4 Данные 1968-2019 гг.}

За 52 года измерения ОМП выполнены семью обсерваториями: $\mathrm{KpAO}^{1}$ (Котов, 2013), Маунт Уилсон, $\mathrm{WSO}^{2}$ (Scherrer et al., 1977b), Саяны (Институт солнечно-земной физики, Иркутск; Демидов и др., 2005), Сатерленд (Chaplin et al., 2003), Национальная солнечная обсерватория США (NSO) ${ }^{3}$ и Kисловодск (Пулково). В сумме получено около 28 тыс. суточных значений ОМП, что позволяет наиболее точно определить скорость солнечного вращения и изучить поведение ОМП с 11-летним циклом.

Таблица 1. Измерения ОМП в семи обсерваториях, 1968-2019 гг.

\begin{tabular}{|c|c|c|c|c|c|c|}
\hline Обсерватория & Годы & Линия (нм) & $N$ & $\Delta, \Gamma \mathrm{c}$ & $S, \Gamma \mathrm{c}$ & $k$ \\
\hline $\mathrm{KpAO}$ & $1968-2018$ & Fe I $\lambda 525.02$ & 3890 & 0.11 & 0.61 & 1.00 \\
\hline $\mathrm{KpAO}$ & 2001-2018 & Fe I $\lambda 524.71$ & 1863 & 0.14 & 0.61 & 0.99 \\
\hline Маунт Уилсон & 1970-1982 & Fe I $\lambda 525.02$ & 2457 & 0.07 & 0.67 & 0.90 \\
\hline WSO & 1975-2019 & $"$ & 13368 & 0.05 & 0.37 & 1.64 \\
\hline Саяны & $1982-2015$ & $"$ & 477 & 0.05 & 0.72 & 0.84 \\
\hline Сатерленд & $1992-2001$ & K I $\lambda 769.90$ & 1988 & 0.01 & 0.43 & 1.41 \\
\hline NSO & 2003-2017 & Fe I $\lambda 630.15$ & 3536 & 0.01 & 0.45 & 1.35 \\
\hline Кисловодск & 2014-2015 & Fe I $\lambda 630.25$ & 295 & 0.01 & 0.99 & 0.61 \\
\hline Вместе $^{a}$ & $1968-2019$ & - & 27874 & - & 0.61 & - \\
\hline
\end{tabular}

Список данных приведен в табл. 1 , где $N$ - число суточных значений напряженности $B$ продольного поля видимой солнечной полусферы, $\Delta$ - типичная ошибка отдельного измерения, $S-$ стандартное отклонение массива и $k$ - нормировочный коэффициент, с помощью которого данные сведены в общий ряд 1968-2019 гг. с числом $N=27874, S=0.61$ Гс и средним $-0.010(4)$ Гс; спектральные линии, по которым измерялось поле, указаны в третьем столбце. Положительные $B$ соответствуют северной полярности, нулевая фаза - эпохе 0 UT, 1 января 1968 г., а спектры мощности (CM) или периодограммы, вычислялись сначала методом наложенных эпох, затем - прямым

\footnotetext{
1 http://crao.ru

${ }^{2}$ http://wso.stanford.edu

3 https://solis.nso.edu
} 
Фурье-преобразованием (программы были разработаны Д.Н. Рачковским (1985) и, более совершенные, В.И. Ханейчуком).

\section{5 Вращение Солнца}

В 1968-1976 гг. двухсекторная структура вращалась с синодическим периодом $P=27.03(6)$ сут, но с добавлением данных он приблизился к 26.92(2) сут (Haneychuk et al., 2003). B CM полного 52 летнего ряда соответствующий пик когерентного вращения, однако, отсутствует, см. рис. 1.

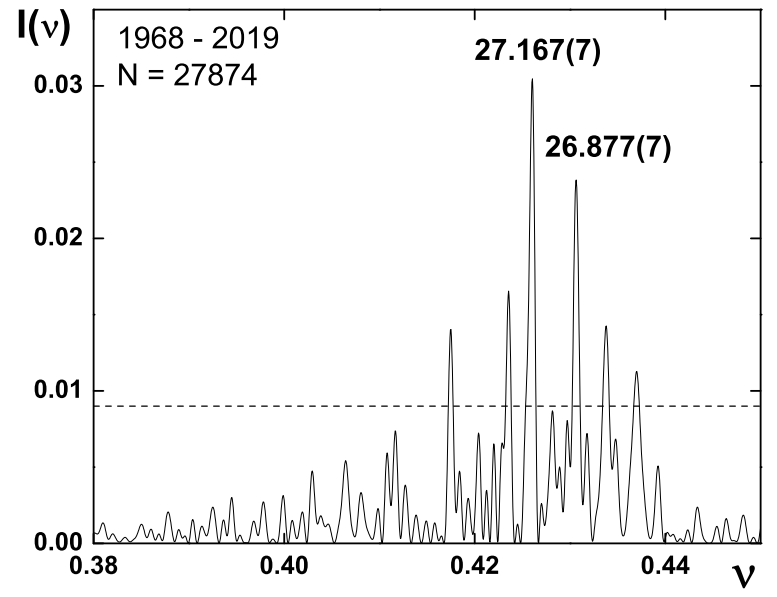

Рис. 1. Спектр мощности ОМП 1968-2019 гг. $(N=27874)$ для частот солнечного вращения. По горизонтали - частота $\nu$ в мкГц, по вертикали мощность $I(\nu)$ в произвольных единицах; пунктирной линией обозначен уровень значимости $3 \sigma$, числами - период в сутках

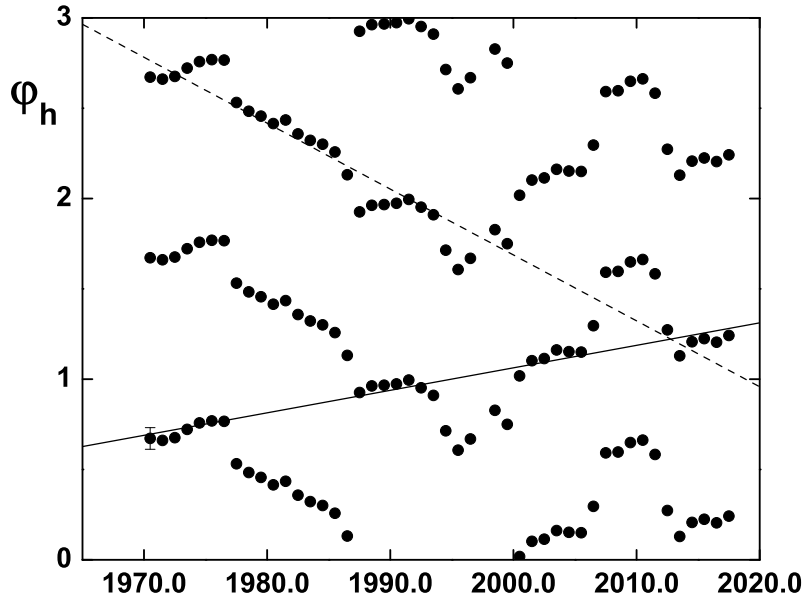

Рис. 2. Диаграмма О-C, построенная с периодом 27.000 сут. По горизонтальной оси - годы, по вертикали - фазы $\varphi_{h}$ (точки, см. текст) с повторением для фазовых интервалов 1-2 и 2-3. Вертикальной черточкой показана типичная неопределенность фазы, а две наклонные линии - прямые регрессий, указывающих на действительные периоды 26.927(7) сут (пунктирная) и 27.025(4) сут (сплошная)

Для определения главных периодов изменения ОМП определялись амплитуды и фазы $\varphi_{h}$ максимума синусоид, построенных с пробным периодом 27.000 сут для каждого последовательного пятилетнего отрезка данных. В результате получена диаграмма О-C (рис. 2), где прямые линейной регрессии говорят о двух периодах: 26.927(7) сут и 27.025(4) сут, с нарушениями и восстановлениями начальных фаз, которые уточнены с помощью соответствующих CM (Kotov, 2019a):

$$
P_{E Q}=26.930(7), \quad P_{\odot}=27.027(6) .
$$

Первый, согласуясь со спектроскопическим периодом 26.94(17) сут (Scherrer et al., 1980), отвечает вращению экватора, второй - вращению гравитирующей массы Солнца как звезды. Соответствующие сидерические периоды равны, в сутках:

$$
P_{E Q}^{\prime}=25.081(7), \quad P_{\odot}^{\prime}=25.165(6)
$$

(интересно, что период их биений, 21.1(2.4) г., совпадает в пределах ошибки с длительностью цикла Хейла, 22.14(8) г.). Видим, что синодическое вращение Солнца как звезды находится в тесных резонансах с орбитальным и осевым движениями Земли: 27:2 и 1:27 соответственно, см. (1).

Действительно, только три временны́е шкалы характеризуют движения системы Солнце-Земля: $P_{\odot}=27.027(6)$ сут, $P_{Y}=365.256$ сут и $P_{D}=1.000$ сут (последние два - орбитальный и осевой, относительно Солнца, периоды движения Земли соответственно). Они отвечают паре безразмерных параметров, больших единицы: 


$$
\frac{P_{Y}}{P_{\odot}}=13.514(3), \quad \frac{P_{\odot}}{P_{D}}=27.027(6),
$$

приводящих к резонансным соотношениям диофантового типа:

$$
2 \frac{P_{Y}}{P_{\odot}} \approx \frac{P_{\odot}}{P_{D}} \approx 27
$$

Левая часть (4), точная до неопределенности $0.01 \%$ периода $P_{\odot}$, говорит, что за один год наша звезда совершает почти 27 полуоборотов, синодических, тогда как Земля за один солнечный оборот - такое же число своих осевых вращений относительно Солнца. Это указывает на скрытую связь между движениями Солнца и Земли: длительности суток и года связаны между собой, что может быть вызвано, например, когерентными колебаниями гравитации внутри Солнечной системы (предмет будущих исследований).

На основе (3) для синодического периода вращения Солнца, в сутках, получаем:

$$
P_{\odot}=\left(2 P_{Y} P_{D}\right)^{1 / 2} \approx 27.028
$$

что согласуется с наблюдаемым значением 27.027(6) сут. (Остается открытым вопрос: случайно ли Луна движется с сидерическим периодом 27.322 сут, близким к соизмеримости с $\left.P_{\odot} ?\right)$

Таким образом, Солнце относительно далеких звезд вращается с периодами $P_{E Q}^{\prime}=25.081(7)$ сут, что соответствует скорости вращения вещества и поля на экваторе (согласие отражает, очевидно, вмороженность поля в плазму), и $P_{\odot}^{\prime}=25.165(6)$ сут - для всего Солнца. С Земли, однако, мы регистрируем экваториальный период 26.930(7) сут, половина которого, 13.465(4) сут, совпадает в пределах ошибки с периодом солнечных "магнитных колебаний" 13.4582(25) сут - главного, когерентного на протяжении десятилетий периода 4-секторной структуры ОМП. Эта величина согласуется также с динамической, или резонансной, шкалой 13.4577(10) сут планетной системы (шкала имеет, по-видимому, гравитационную природу; Kotov, 2018).

На основе (5) для теоретического орбитального периода Земли получаем в сутках:

$$
P_{Y} \approx \frac{P_{\odot}^{2}}{2 P_{D}}=365.23(17),
$$

что согласуется с наблюдениями. Но поскольку по закону Ньютона

$$
P_{Y}=2 \pi\left(\frac{a^{3}}{G M_{\odot}}\right)^{1 / 2}
$$

(обозначения обычные, $a=1$ а. е.), для гравитационной постоянной, приравняв (6) и (7), получаем:

$$
G=\frac{a^{3}}{M_{\odot}}\left(4 \pi \frac{P_{D}}{P_{\odot}^{2}}\right)^{2}=6.675(6)
$$

в единицах $10^{-8} \mathrm{~cm}^{3} \Gamma^{-1} \mathrm{c}^{-2}$. Это согласуется со значением 6.67408(31) Tanabashi et al. (2019) и еще лучше - с недавним лабораторным измерением 6.67554(16) Quinn et al. (2014), а также с "космической” величиной 6.67543(2), полученной Саншез и др. (2013) из симметрии трех фундаментальных взаимодействий: электромагнитного, гравитационного и слабого (и на основе наблюдений глобальных пульсаций Солнца с периодом 9600.606(12) с; см. Severny et al., 1976; Котов, Ханейчук, 2016). Согласие, однако, пропадает, если $P_{D}$ и $P_{\odot}$ в $(8)$ заменить сидерическими величинами; объяснение этой загадки - в будущих исследованиях ОМП и динамики Солнечной системы.

\section{6 Циклы 22 года и 7 лет}

В низкочастной части $\mathrm{CM}$ на рис. 3 наиболее примечательные пики отвечают периодам $P_{C}=$ 20.4(1.4) г. и $P_{7}=7.12(17)$ лет (для частот $\nu \lesssim 0.015$ мкГц фактический уровень $3 \sigma$, отвечая "красному" шуму $I(\nu) \sim \nu^{-1}$, расположен выше пунктирной линии). Если первый соответствует, почти в пределах ошибки, циклу Хейла $P_{H}=22.14(8)$ г., или удвоенному циклу Вольфа $P_{W}=11.07(4)$ г., то второй - неизвестной природы (об остальных пиках, превышающих уровень значимости $3 \sigma$, см. Kotov, 2019b). 


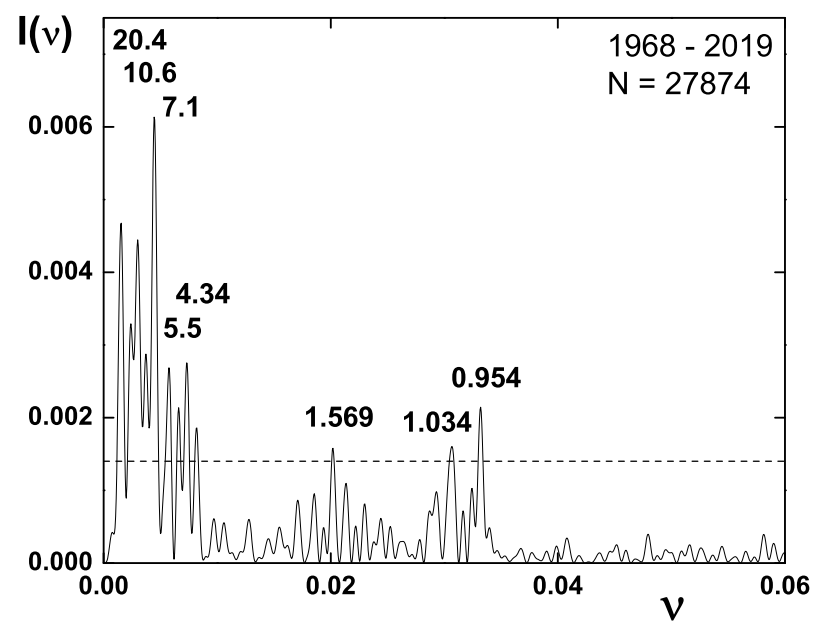

Рис. 3. Спектр мощности ОМП за 1968-2019 гг. $(N=27874)$. По горизонтали - частота $\nu$ в мкГц, по вертикали - мощность $I(\nu)$ в произвольных единицах; пунктирная линия соответствует значимости $3 \sigma$, а числами обозначены наиболее заметные пики (период в годах)

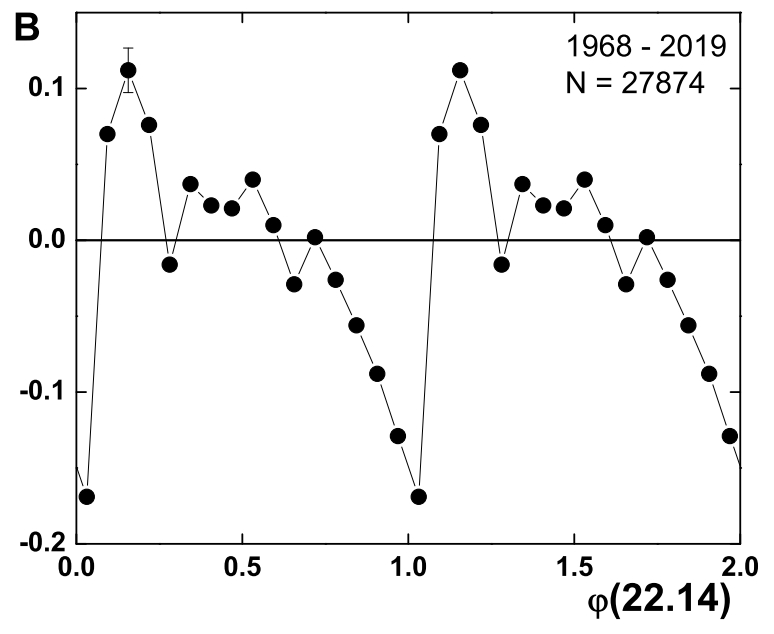

Рис. 4. Средняя кривая изменения ОМП с периодом Хейла 22.14 г. $(N=27874)$. По горизонтали фаза $\varphi$, по вертикали - напряженность $B$ в Гс; вертикальной черточкой указана типичная стандартная ошибка для каждого из 16 блоков данных

Кривая изменения ОМП с периодом $P_{H}$, приведенная на рис. 4, имеет негармонический профиль, что стимулировало обсуждение гипотезы о "несолнечной", или космической, природе цикла (без отрицания эффективности механизма динамо для проявлений цикла в фотосфере и конвективной зоне; см. Котов и Саншез, 2017). Средняя кривая 7-летнего периода, не приведенная здесь, близка к синусоиде с амплитудой $\approx 0.08$ Гс.

После уточнения этого периода $P_{7}=7.06(13)$ г. к нему возникает особый интерес: $(a)$ соответствующий пик в СМ по высоте превышает пик $P_{C}$ и (б) отношение цикла Хейла к $P_{7}$ составляет $3.14(6)$, т. е., в пределах ошибки, мировую константу $\pi$. Известно также, что некоторые периодические процессы в природе связаны с числом $\pi$ как фактором геометрии пространства; именно поэтому $\pi$ входит, например, в формулу вероятности (подчеркнем, что отношение $P_{H} / P_{7}$ несколько ближе к $\pi$, чем к тройке, со значимостью отличия $2.3 \sigma)$.

Следующий шаг - признание, что $\pi$ может характеризовать не только пространство, но и время, проявляясь, например, в устойчивости определенных периодических процессов по отношению к другим, более фундаментальным временны́м масштабам, процессам и циклам на Солнце и во Вселенной. Таким примером по отношению к $P_{H}$ и может служить 7 -летний цикл ОМП.

\section{7 Космическая природа циклов?}

Пекулярный вид кривой $P_{H}$ на рис. 4 наводит на мысль, что в основе цикла Хейла (с "половинным" значением - циклом Вольфа $\left.P_{W}\right)$ лежит нелинейный механизм, вряд ли механизм динамо, и что сам цикл имеет космическое происхождение. Кривая "магнитного" цикла подтверждает также правило Гневышева и Оля (1948): цикл Хейла состоит из двух 11-летних, начинаясь с четного цикла Вольфа, с меньшим числом пятен в максимуме.

Указание на космологическое происхождение 22-летнего цикла следует также из анализа эпох экстремумов чисел Вольфа (Котов и др., 2012). Постоянство начальной фазы - со времен Галилея говорит, что не столько в глубине Солнца скрыты некие “часы” (управляющие, по Дикке (1978), ходом цикла), сколько сам цикл имеет экзогенную природу. Согласно Sanchez et al. (2011), солнечный 11-летний цикл - отражение некоего периодического свойства наблюдаемой Вселенной, что созвучно с утверждением “... 11-летний солнечный цикл, вероятно, является самым известным квазипериодическим явлением на Солнце, а может быть, и в астрофизике вообще" (Обридко, 2008). (Уточним: самые известные и наиболее "древние" периодические явления в астрономии связаны с сутками, годом и месяцем, т. е. с движениями системы Земля-Луна. И уже показано (Kotov, 2019a), что 
связь солнечного вращения с движениями Земли, определяющими длительности года и суток, - a также, по-видимому, движениями Луны, - неслучайная; это заставляет более серьезно отнестись к антропному принципу, см. Carr, Rees, 1979; Рубаков, Штерн, 2020.)

Легко найти следы "солнечного" цикла на "квантовых границах" Вселенной. Действительно, недавно было найдено голографическое соотношение между длиной Хейла $L_{H} \equiv c P_{H}$ и размерами Вселенной и водородного атома:

$$
P_{H} \equiv \frac{L_{H}}{c} \approx 2 \frac{\left(a_{B} R_{U}^{3}\right)^{1 / 4}}{c}=22.03(5),
$$

в годах, где $a_{B}=\hbar^{2} / m_{e} e^{2} \approx 0.529177 \times 10^{-8}$ см и $R_{U}=c T_{U}=1.306(4) \times 10^{28}$ см - радиусы Бора и Вселенной соответственно, а $T_{U}=13.80(4) \times 10^{9}$ г. - "возраст" Вселенной (см. Котов и др., 2012; Kotov, Sanchez, 2017). Выражение (9) нельзя воспринимать как результат игры в нумерологию, т. к. оно имеет простое геометрическое истолкование:

$$
\frac{L_{W}}{a_{B}} \approx\left(\frac{R_{U}}{L_{W}}\right)^{3},
$$

где длина волны Вольфа $L_{W} \equiv L_{H} / 2$, выраженная в радиусах атома водорода, с точностью $2 \%$ равна объему Вселенной, выраженному в объемах сферы Вольфа радиуса $L_{W}$.

В соответствии с (9) для теоретического 7-летнего периода получаем в годах:

$$
P_{7} \approx 2 \frac{\left(a_{B} R_{U}^{3}\right)^{1 / 4}}{\pi c}=7.01(2) .
$$

Интерес вызывает и формальная связь орбитального периода Земли с колебанием $P_{7}$ :

$$
P_{Y}=(\pi-3) P_{7},
$$

равенство, точное до $0.04 \%$, что меньше неопределенности $P_{7}$; его смысл еще надо выяснять.

\section{8 Заключение}

Солнце в пределах ошибок измерений - сферической формы, причем $R_{\odot}$ и $L_{\odot}$ практически неизменны на масштабах месяцы-годы (слабые вариации $L_{\odot}$, обусловленные вращением, активными процессами, 11-летним циклом, пятнами и другими образованиями фотосферы, легко объясняются). Влияние же планет на Солнце было отвергнуто в ХХ в. из-за ничтожности соответствующих гравитационных возмущений по сравнению с тяжестью самого Солнца. Поэтому и возникает интерес к изменениям ОМП, обусловленным движением электрических зарядов. А именно, при взаимодействии элементарных частиц - например, электрона и протона - эффективность электрической силы $F_{e}$ на много порядков величины превосходит взаимодействие гравитационное с силой $F_{g}$ :

$$
\frac{F_{e}}{F_{g}}=\frac{e^{2}}{G m_{e} m_{p}} \approx 2 \times 10^{39} .
$$

Поэтому возмущения со стороны планет способны породить существенные вариации систем глобальных электротоков и, следовательно, переменность ОМП. И главная причина - не только природа двух сил, но и большая разность масс $m_{e}$ и $m_{p}$, ведущая к различию соответствующих сил торможения, причем амплитуда эффектов может усиливаться благодаря резонансным, плохо изученным механизмам.

Здесь подходим к Солнцу как “звездному магниту" (см. Gough, 2017): многолетние наблюдения ОМП дают возможность не только взглянуть вглубь звезды, но и лучше понять динамику Солнечной системы. А в связи с глобальными пульсациями Солнца (с периодом 9600 с неизвестной природы; см. Kotov, Haneychuk, 2020), указанными выше резонансами системы Солнце-Земля и имея ввиду, в частности, теорию приливной синхронизации солнечного динамо (Stefani et al., 2019; Scafetta, 2020), разумно полагать, что модель Солнца должна быть улучшена.

Цикл $P_{7}$ может быть объяснен двояко: (1) обертон цикла Хейла, обусловленный пилообразной формой профиля последнего, или (2) источник "скрытого" цикла более глубокий: $P_{7}-$ продукт "закона троек"; сам же закон имеет фундаментальный характер, связанный с центральной симметрией 
(пространства и самого Солнца). Придерживаясь (2) и подчеркивая, что $P_{7}$ в $\pi$ раз короче, чем $P_{H}$, мы трактуем его как скрытый период несоизмеримости, обусловленный геометрией пространства. Корни его кроются, по-видимому, в появлении $\pi$ в интеграле вероятности (Горобец, 2004), потому что не только физические процессы, но и все математические операции и наши наблюдения производятся в мире, обладающем пространственной симметрией.

Биения с циклом Хейла 22.14 г. процессов, обусловленных как циклом $P_{7}$, так и утроенным периодом $3 P_{7} \approx 21.1$ г., ведут, возможно, к нарушениям когерентности 11-летнего цикла Вольфа и 22-летнего - Хейла, а также к более длительным циклам Солнца (приводящим, гипотетически, к климатическим процессам на Земле и связанным, возможно, с другими явлениями в Солнечной системе). Физический механизм появления мировой константы $\pi$ у Солнца, однако, еще предстоит раскрыть. Для объяснения $P_{7}$ полезно, наверное, привлечь другие модели Солнца, отличные от стандартной: один и тот же физический объект или процесс, в принципе, может быть описан разными моделями (например, фотон - или волна, или частица). Высокоточные и регулярные измерения ОМП могут дать также новый ключ к объяснению уникальности Земли и долговременной стабильности Солнечной системы - загадки, которую безуспешно решали Ньютон, Лаплас и другие титаны прошлого.

Я с благодарностью и теплотой вспоминаю годы совместной работы и горячие беседы с акад. А.Б. Северным, а также с инженером-гением Н.С. Никулиным и техником A.М. Чижовым (оба - ветераны Великой Отечественной войны, что отмечаю особо в год 75-летия Победы и 75-летия КрАО); признателен И.А. Егановой и Ф.М. Саншезу (Париж) за многочисленные дискуссии о свойствах Солнца и Космоса, устройстве Солнечной системы и природе физических законов, В.И. Ханейчуку за активное участие в наблюдениях Солнца в $\mathrm{KpAO,} \mathrm{наблюдателям} \mathrm{других} \mathrm{обсерваторий} \mathrm{за} \mathrm{их} \mathrm{ОМП-данные} \mathrm{и} \mathrm{рецензен-}$ ту за полезные замечания. Благодарю также судьбу за возможность общения со многими другими выдающимися исследователями Солнца и Вселенной, среди которых Дж. Уилкокс, Ф. Шеррер, Х.И. Абдусаматов, Дж. Айзек, Г. ван дер Рей, Ю.И. Витинский, Р. Говард, Д. Гоф, М.Л. Демидов, Е.В. Иванов, Р.Н. Ихсанов, В.М. Кувшинов, Г.В. Куклин, П.Г. Куликовский, С. Кучми, У. Ливингстон, В.М. Лютый, Д.Я. Мартынов, Н.С. Нестеров, В.Н. Обридко, ЖК.К. Пекер, Л. Свалгард, Я. Стенфло, Э. Фосса, Г. Хилл, Дж. Хэксма, Л.И. Цветков, Н.С. Черных, Ю. Штауде и др.

\section{Литература}

Гневышев М.Н., Оль А.И., 1948. Астрон. журн. Т. 25. № 1. С. 18. [Gnevyshev M.N., Ohl A.I., 1948. Astron. zhurn., vol. 25, no. 1, p. 18. (In Russ.)]

Горобец Б., 2004. Наука и жизнь. № 2. С. 64. [Gorobets B., 2004. Nauka i zhizn, no. 2, p. 64. (In Russ.)] Демидов М.Л., Григорьев В.М., Пещеров В.С., 2005. Астрон. журн. Т. 82. № 7. С. 628. [Demidov M.L., Grigor'ev V.M., Peshcherov V.S., 2005. Astron. zhurn., vol. 82, no. 7, p. 628. (In Russ.)]

Котов В.А., 2007. Изв. Крымск. Астрофиз. Обсерв. Т. 103. № 2. С. 245. [Kotov V.А., 2007. Izv. Krymsk. Astrofiz. Observ., vol. 103, no. 2, p. 245. (In Russ.)]

Котов В.А., 2013. Изв. Крымск. Астрофиз. Обсерв. Т. 109. № 1. С. 232. [Kotov V.A., 2013. Izv. Krymsk. Astrofiz. Observ., vol. 109, no. 1, p. 232. (In Russ.)]

Котов В.А., Саншез Ф.М., Бизуар К., 2012. Изв. Крымск. Астрофиз. Обсерв. Т. 108. № 1. С. 57. [Kotov V.A., Sanshez F.M., Bizuar K., 2012. Izv. Krymsk. Astrofiz. Observ., vol. 108, no. 1, p. 57. (In Russ.)]

Котов В.А., Северный А.Б., 1983. Общее магнитное поле Солнца как звезды. Каталог 1968-1976. М.: AH CCCP. [Kotov V.A., Severnyi A.B., 1983. The Total Magnetic Field of the Sun as a Star, Catalog 1968-1976. Moscow: Akad. Nauk SSSR. (In Russ.)]

Котов В.А., Северный А.Б., Цап Т.Т., 1982. Изв. Крымск. Астрофиз. Обсерв. Т. 65. С. 3. [Kotov V.A., Severnyi A.B., Tsap T.T., 1982. Izv. Krymsk. Astrofiz. Observ., vol. 65, p. 3. (In Russ.)]

Котов В.А., Ханейчук В.И., 2016. Изв. Крымск. Астрофиз. Обсерв. Т. 112. С. 125. [Kotov V.A., Haneychuk V.I., 2016. Izv. Krymsk. Astrofiz. Observ., vol. 112, p. 125. (In Russ.)]

Никулин Н.С., Северный А.Б., Степанов В.Е., 1958. Изв. Крымск. Астрофиз. Обсерв. Т. 19. С. 3. [Nikulin N.S., Severnyi A.B., Stepanov V.E., 1958. Izv. Krymsk. Astrofiz. Observ., vol. 19, p. 3. (In Russ.)] 
Обридко В.Н., 2008. Плазменная гелиофизика. Т. 1. М.: Физматлит. [Obridko V.N., 2008. Plasma Heliophysics, vol. 1. Moscow: Fizmatlit. (In Russ.)]

Рачковский Д.Н., 1985. Изв. Крымск. Астрофиз. Обсерв. Т. 71. С. 25. [Rachkovskiy D.N., 1985. Izv. Krymsk. Astrofiz. Observ., vol. 71, p. 25. (In Russ.)]

Рубаков В.А., Штерн Б.Е., 2020. Земля и Вселенная. № 1. С. 27. [Rubakov V.А., Shtern B.E., 2020. Earth \& Universe, no. 1, p. 27. (In Russ.)]

Саншез Ф.М., Котов В.А., Бизуар К., 2013. Изв. Крымск. Астрофиз. Обсерв. Т. 109. № 3. С. 181. [Sanshez F.M., Kotov V.A., Bizuar K., 2013. Izv. Krymsk. Astrofiz. Observ., vol. 109, no. 3, p. 181. (In Russ.)]

Северный А.Б., 1955. Изв. Крымск. Астрофиз. Обсерв. Т. 15. С. 31. [Severny А.В., 1955. Izv. Krymsk. Astrofiz. Observ., vol. 15, p. 31. (In Russ.)]

Северный А.Б., Кувшинов В.М., Никулин Н.С., 1974. Изв. Крымск. Астрофиз. Обсерв. Т. 50. С. 3. [Severny A.B., Kuvshinov V.M., Nikulin N.S., 1974. Izv. Krymsk. Astrofiz. Observ., vol. 50, p. 3. (In Russ.)]

Carr B.J., Rees M.J., 1979. Nature, vol. 278, p. 605.

Chaplin W.J., Dumbill A.M., Elsworth Y., et al., 2003. Mon. Not. Roy. Astron. Soc., vol. 343, p. 813.

Dicke R.H., 1978. Nature, vol. 276, p. 676.

Gough D.O., 2017. Solar Phys., vol. 292, p. 70.

Haneychuk V.I., Kotov V.A., Tsap T.T., 2003. Astron. Astrophys., vol. 403, p. 1115.

Kotov V.A., 2018. Astron. Nachr., vol. 339, no. 6, p. 513.

Kotov V.A., 2019a. Adv. Space Res., vol. 63, p. 3385.

Kotov V.A., 2019b. Astrophys. Space Sci., vol. 364, no. 3, p. 45.

Kotov V.A., Haneychuk V.I., 2020. Astron. Nachr., doi:10.1002/asna.202013797.

Kotov V.A., Sanchez F.M., 2017. Astrophys. Space Sci., vol. 362, no. 1, p. 6.

Quinn T., Speake C., Parks H., Davis R., 2014. Phil. Trans. R. Soc. A, vol. 372, p. 20140032.

Sanchez F.M., Kotov V.A., Bizouard C., 2011. J. Cosmology, vol. 17, p. 7225.

Scafetta N., 2020. Solar Phys., vol. 295, no. 2, p. 33, doi:10.1007/s11207-020-1599-y.

Scherrer P.H., Wilcox J.M., Kotov V., Severny A.B., Howard R., 1977a. Solar Phys., vol. 52, p. 3.

Scherrer P.H., Wilcox J.M., Svalgaard L., 1980. Astrophys. J., vol. 241, p. 811.

Scherrer P.H., Wilcox J.M., Svalgaard L., et al., 1977b. Solar Phys., vol. 54, p. 353.

Severny A., 1969. Nature, vol. 224, p. 53.

Severny A.B., Kotov V.A., Tsap T.T., 1976. Nature, vol. 259, p. 87.

Stefani F., Giesecke A., Weier T., 2019. Solar Phys., vol. 294, p. 60.

Tanabashi M., Hagiwara K., Hikasa K., et al., 2019. Phys. Rev. D, vol. 98, p. 030001. 


\title{
Crimean observations of the magnetic Sun: 1967-2018
}

\author{
V.A. Kotov
}

Crimean Astrophysical Observatory, Nauchny 298409

vkotov@craocrimea.ru

\begin{abstract}
The magnetic field measurements of the Sun as a star initiated by academician A.B. Severny have been supported by six other observatories. The history of such investigations at $\mathrm{CrAO}$ and the basic results are briefly described. The synodic spin period of the gravitating solar mass $P_{\odot}=27.027(6)$ days is determined; the latter is shown to be linked to the Earth's motion: the Sun performs 27 semi-revolutions over one terrestrial year, and the Earth - the same number of its revolutions with period $P_{D}$ during one full solar rotation. The field is also changing with the Hale cycle $P_{H} \approx 22$ years and the cycle $P_{7}=7$ years, whereas their ratio coincides with the Archimedes approximation, 22:7, for the $\pi$ number, the timescale $(\pi-3) P_{7}=P_{\odot}^{2} / 2 P_{D}$ - with the Earth's orbital period. We provide arguments in favour of the cosmic origin of both cycles and holographic expressions, including $P_{H}, P_{7}, \pi$, and universal constants.
\end{abstract}

Key words: CrAO history, Sun, magnetic field, rotation, 11-year cycle 